\title{
Effects of fish oil supplementation in rheumatoid arthritis
}

Hille van der Tempel, Jacob E Tulleken, Pieter C Limburg, Frits A J Muskiet, Martin H van Rijswijk

\begin{abstract}
Sixteen patients with rheumatoid arthritis entered a trial to determine the clinical and biochemical effects of dietary supplementation with fractionated fish oil fatty acids. A randomised, double blind, placebo controlled crossover design with 12 week treatment periods was used. Treatment with nonsteroidal anti-inflammatory drugs and with disease modifying drugs was continued throughout the study. Placebo consisted of fractionated coconut oil. The following results favoured fish oil rather than placebo: joint swelling index and duration of early morning stiffness. Other clinical indices improved but did not reach statistical significance. During fish oil supplementation relative amounts of eicosapentaenoic acid and docosahexaenoic acid in the plasma cholesterol ester and neutrophil membrane phospholipid fractions increased, mainly at the expense of the omega-6 fatty acids. The mean neutrophil leucotriene $B_{4}$ production in vitro showed a reduction after 12 weeks of fish oil supplementation. Leucotriene $B_{5}$ production, which could not be detected either in the control or in the placebo period, rose to substantial quantities during fish oil treatment. This study shows that dietary fish oil supplementation is effective in suppressing clinical symptoms of rheumatoid arthritis.
\end{abstract}

Polyunsaturated fatty acids play an important part in the structural and metabolic function of cellular membranes. One of the major polyunsaturated fatty acids is arachidonic acid. Oxygenation of arachidonic acid leads to the production of potent mediators of inflammation. ${ }^{1-3}$ Alteration of dietary fatty acid composition may result in competition with the incorporation of arachidonic acid into the cell membrane and thereby modulate the functional qualities of the cell.

Fatty acids commonly consumed in the Western diet-for example, linoleic acid-are converted via arachidonic acid into cyclooxygenase metabolites of the 2 series, whereas leucotrienes of the 4 series are produced via the lipoxygenase pathway. Leucotrienes are potent lipid mediators, which play an important part in allergic and inflammatory reactions. ${ }^{4-6}$ Leucotriene $\mathrm{B}_{\mathbf{4}}$, mainly produced in neutrophils, is a strong leucocyte activator. Its effects are comparable with C5a, f-Met-Leu-Phe, and platelet activating factor and include chemotaxis, nondirected migration, aggregation, and lysosomal enzyme release. ${ }^{7-12}$
Polyunsaturated fatty acids with three carbon atoms from the terminal methyl group to the first double bond are designated omega- 3 or $n-3$ fatty acids. Eicosapentaenoic acid (20:5, n-3) and docosahexaenoic acid (22:6, n-3) are the main long chain $n-3$ fatty acids in fish oil. Eicosapentaenoic acid incorporated in cell membrane can be converted, via the cyclooxygenase pathway, into prostaglandins, thromboxanes, and prostacyclins of the 3 series. Via the lipoxygenase pathway leucotrienes of the 5 series, in particular leucotriene $\mathrm{B}_{5}$, are produced. ${ }^{13-15}$ Leucotriene $B_{5}$ is 10 to 30 -fold less potent than leucotriene $B_{4}$ in assays of leucocyte function. ${ }^{16} 17$ Thus dietary fish oil supplementation has potential anti-inflammatory effects owing to the production of functionally attenuated eicosapentaenoic acid derived analogues of the arachidonic acid metabolites. $^{18-21}$ In this paper we describe the clinical and biochemical effects of dietary fatty acid supplementation with fish oil in patients with rheumatoid arthritis.

\section{Patients and methods}

PATIENTS

Sixteen patients (nine female, seven male, mean age 53 years, mean duration of disease 12 years) with classical or definite rheumatoid arthritis were admitted to the study (table 1). Fifteen of them received non-steroidal anti-inflammatory drugs. Eleven patients received disease modifying drugs (gold, antimalarial drugs, and Dpenicillamine) in addition. None of the patients received steroids or cytostatic drugs.

\section{STUDY DESIGN}

We used a double blind, placebo controlled, crossover design with 12 week treatment periods. Patients were randomly allocated to receive each day either 12 capsules of fractionated fish oil or fractionated coconut oil, flavoured with fish aroma, as placebo (Intradal, Amersfoort, The Netherlands). The fish oil capsules held a majority of $20: 5, \mathrm{n}-3(31 \mathrm{~mol} / 100 \mathrm{~mol}$ fatty acids $(\mathrm{mol} \%)$ ) and $22: 6, \mathrm{n}-3(22 \mathrm{~mol} \%)$, resulting in a

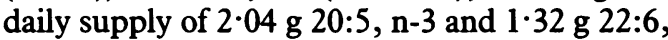
$\mathrm{n}-3$, whereas the coconut oil capsules mainly contained C8:0 (63 mol\%) and $\mathrm{Cl0:0}$ (36 mol\%). A 12 week run-in period without fatty acid supplementation was used to confirm stable disease activity. At week 13 supplementation with either fish oil or placebo started. After 12 weeks the patients crossed over from fish oil to placebo or vice versa. Patients and doctors were blinded to treatment assignment
9700 RB Groninge

Accepted for publication

15 June 1989 
Table 1: Clinical and biochemical disease variables before and after three months of fish oil and coconut oil treatment. Values are expressed as means (SEM), unless otherwise indicated

\begin{tabular}{|c|c|c|c|}
\hline \multirow{2}{*}{ Disease variables } & \multicolumn{3}{|l|}{ Supplementation } \\
\hline & None & Fish oil & Coconut oil \\
\hline 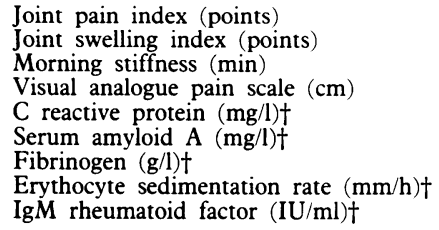 & $\begin{array}{ll}33 & (7) \\
10 & (2) \\
46 & (15) \\
3 \cdot 1 & (0 \cdot 6) \\
30 & (3-121) \\
14 & (1-100) \\
4 \cdot 4 & (2 \cdot 6-7 \cdot 3) \\
30 & (19-98) \\
130 & (3-1050)\end{array}$ & $\begin{array}{ll}29 & (7) \\
2 & (1) \\
15 & (5) \\
2 \cdot 7 & (0 \cdot 5) \\
17 & (3-69) \\
14 & (1-127) \\
4 \cdot 3 & (2 \cdot 4-5 \cdot 6) \\
34 & (14-80) \\
100 & (3-850)\end{array}$ & $\begin{array}{ll}42 & (9) \\
8 & (3) \\
50 & (13) \\
4 & (0 \cdot 7) \\
21 & (5-71) \\
28 & (1-95) \\
4 \cdot 3 & (2 \cdot 9-5 \cdot 3) \\
40 & (11-70) \\
165 & (3-900)\end{array}$ \\
\hline
\end{tabular}

during the entire study. The patients were instructed to continue their regular drug treatment schedule. Patients' dietary fat intake was kept constant throughout the study. Each patient kept a daily food diary. Every two weeks the patients were observed by the same rheumatologist, biometrist, and dietician. The study was approved by the local medical ethical committee and informed consent was obtained from all patients.

\section{CLINICAL EVALUATION}

For clinical evaluation the following were monitored: duration of early morning stiffness (in minutes), joint pain (on a four point scale: 0 =absent, $1=$ mild, 2 =moderate, $3=$ severe), the joint swelling index (on a three point scale: 0 =absent, $1=$ moderate, $2=$ severe), and visual analogue pain scale score on a 10 point scale (from $0=$ no pain to $10=$ worst ever). Grip strength was measured with a manometer $(\mathrm{kPa})$.

\section{LABORATORY EVALUATION}

Laboratory evaluation of peripheral blood indices was carried out every two weeks and comprised a complete blood cell count, erythrocyte sedimentation rate, and plasma concentrations of $C$ reactive protein, fibrinogen, serum amyloid $\mathrm{A}$, and IgM rheumatoid factor.

\section{ASSESSMENT OF IN VITRO PRODUCTION OF LEUCOTRIENES}

Neutrophils were isolated from $30 \mathrm{ml}$ peripheral blood drawn into EDTA. Erythrocytes were sedimented with gelatin $(3 \mathrm{~g} / \mathrm{l})$. The buffy coat was separated on gelatin $(3 \mathrm{~g} / \mathrm{l})$, and neutrophils were subsequently isolated on Percoll $(\mathrm{d}=1 \cdot 077$ $\mathrm{g} / \mathrm{ml}$ ). Residual erythrocytes were lysed with hypotonic ammonium chloride Neutrophils $\left(10^{7}\right.$ cells $/ \mathrm{ml}$ ) were preincubated in Dulbecco's medium for 30 minutes at $37^{\circ} \mathrm{C}$. Subsequently, calcium chloride was added to a final concentration of $2 \mathrm{mmol} / \mathrm{l}$ and the cells were stimulated with $10 \mu \mathrm{M}$ calcium ionophore A23187 for 10 more minutes, then centrifuged at $11000 \mathrm{~g}$ for one minute. Finally, prostaglandin $\mathrm{B}_{2}$ was added to the supernatant as an internal standard for high performance liquid chromatography measurements. Leucotrienes were separated and measured with a $\mathrm{C} 18$ column. ${ }^{22}$
DETERMINATION OF OIL SUPPLEMENT FATTY ACID COMPOSITION

Analyses of both medium and long chain fatty acids in the fish oil and coconut oil capsules were performed by capillary gas chromatography. ${ }^{23}$

DETERMINATION OF PLASMA CHOLESTEROL ESTER FATTY ACID COMPOSITION

After preparation of a plasma total lipid extract and isolation of the cholesterol ester fraction by aminopropyl-silica columns its fatty acid composition was determined by capillary gas chromatography. ${ }^{24}$

DETERMINATION OF NEUTROPHIL PHOSPHOLIPID FATTY ACID COMPOSITION

Neutrophil phospholipids were extracted with chloroform/methanol. Phospholipid subclasses were isolated by high performance liquid chromatography, essentially as described previously. 2526 Their fatty acid composition was determined by capillary gas chromatography $^{27}$ using an apolar stationary phase.

\section{STATISTICAL ANALYSES}

Data were analysed by standard methods based on Student's $t$ test. ${ }^{28}$ For basic treatment comparisons the randomisation $\mathrm{p}$ values were calculated. ${ }^{29}$ To ensure that the overall significance level did not exceed $5 \%$ (one sided) the Bonferroni method was applied to correct for multiple tests. The $\mathrm{p}$ value for each of the response variables was considered significant if less than $1 \%$ (one sided). We also calculated $90 \%$ confidence limits for mean treatment effects: based on the conventional $t$ test and without the Bonferroni correction. Relations between the response variables and also between treatment effects on these variables were studied by the Spearman correlation coefficient.

\section{Results}

Sixteen patients entered the trial. Two patients had to discontinue the study, one because of severe headaches during fish oil supplementation, the second because of gastrointestinal bleeding due to a nicoumalone overdose. During both placebo and fish oil treatment only 
minor, short term side effects were observed (nausea, ructus, and diarrhoea). Most patients endured the fatty acid supplementation without any problems.

During the run-in period no significant changes in clinical or biochemical indices of disease activity were found. As there was no washout period between the two three-month treatment periods the data were analysed to determine whether there were treatment sequence effects. A hangover effect was perceived in most clinical and laboratory variables at four weeks after fish oil treatment was stopped. Yet at treatment end points the changes in clinical and laboratory variables appeared to be independent of treatment sequence.

\section{CLINICAL EVALUATION}

Comparisons were made between end points of fish oil and placebo treatment (table 1). We observed a mean decrease of six points in the joint swelling index $(p=0.01)$ after 12 weeks of fish oil treatment. The duration of morning stiffness decreased by a mean of 35 minutes $(p<0.05)$ during fish oil treatment. The joint pain index showed a trend towards improvement during fish oil supplementation but did not reach statistical significance. Grip strength and visual analogue pain scale score were not altered.

\section{LABORATORY EVALUATION}

Table 1 shows median (range) concentrations at baseline and after 12 weeks of fish oil or placebo supplementation, of plasma fibrinogen, C reactive protein, serum amyloid $\mathrm{A}$, and $\operatorname{IgM}$ rheumatoid factor and the erythrocyte sedimentation rate. The complete blood cell count was not significantly altered. Table 2 shows the differences between neutrophil leucotriene $B_{4}$ production at baseline and after 12 weeks of fish oil and coconut oil supplementation. Mean values decreased from a baseline production of $149 \mathrm{ng} / 10^{7}$ neutrophils to $123 \mathrm{ng} / 10^{7}$ neutrophils $(\mathrm{p}<0.05)$ and leucotriene $\mathrm{B}_{5}$ production increased from undetectable amounts to a mean production of $13 \mathrm{ng} / 10^{7}$ neutrophils $(\mathrm{p}<0.01)$.

Differences between placebo and fish oil treatment in the joint pain index correlated with the differences in the neutrophil leucotriene $B_{4}$ production rates (Spearman correlation coefficient $r=0.90)$. Changes in the other clinical
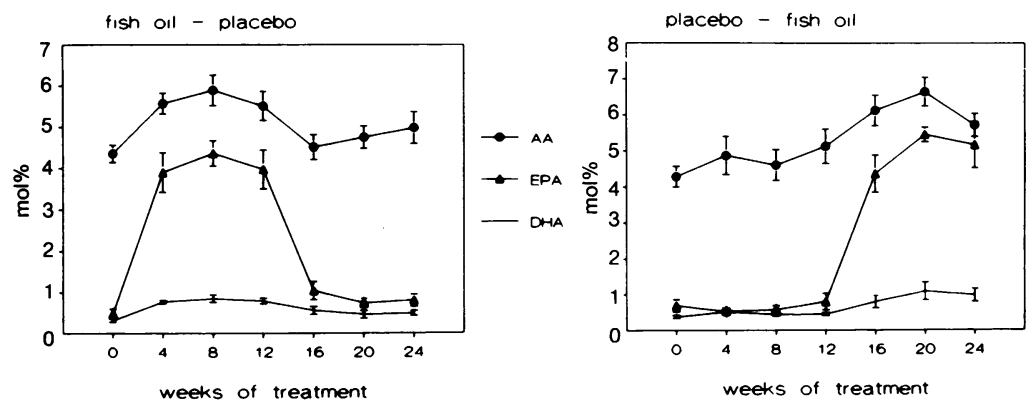

Figure 1: Treatment sequence effects (fish oil-placebo and placebo-fish oil) on the relative composition of selected fatty acids in the plasma cholesterol ester fraction. Treatment of patients was crossed over after 12 weeks. Values are expressed as means (SEM) $A A=$ arachidonic acid; EPA=eicosapentaenoic acid; DHA=docosahexaenoic acid. mol\% $=$ moll $100 \mathrm{~mol}$ fatty acids.
Table 2: In vitro leucotriene production (ng/10 $0^{7}$ neutrophils, $10 \mathrm{~min}$ ) by neutrophils isolated from the blood of patients with rheumatoid arthritis before and after three months of dietary fatty acid supplementation with fish oil or coconut oil. Values are means (SEM)

\begin{tabular}{lll}
\hline Supplementation & \\
\cline { 2 - 3 } None & Fish oil & $\begin{array}{l}\text { Coconut } \\
\text { oil }\end{array}$ \\
\hline
\end{tabular}

\begin{tabular}{lccc}
\hline Leucotriene $\mathrm{B}_{4}$ & $149(13)$ & $123(10)^{*}$ & $141(12)$ \\
Leucotriene $\mathrm{B}_{5}$ & $0(0)$ & $13(2)^{* *}$ & $0(0)$
\end{tabular}

${ }^{*} \mathrm{p}<0.05$, one sided, significantly different from baseline values. $90 \%$ Confidence limits $=6-46$ for mean treatment effect. ${ }^{* *} \mathrm{p}<0.01$, one sided, significantly different from baseline and coconut oil values. $90 \%$ Confidence limits $=9-17$ for mean treatment effect.

variables did not correlate with changes in the leucotriene production.

Within four to eight weeks after discontinuation of fish oil supplementation leucotriene $B_{5}$ production had fallen below the detection level again.

PLASMA CHOLESTEROL ESTERS AND NEUTROPHIL MEMBRANE PHOSPHOLIPIDS

In the placebo period no significant alterations were observed in the fatty acid composition of the plasma cholesterol ester fraction. Figure 1 shows the course of the composition of selected fatty acids in the plasma cholesterol ester fraction during the study. The omega- 3 fatty acid incorporation reached a maximum within one month of fish oil administration. In all cases these values persisted during the fish oil period. In the plasma cholesterol ester fraction the omega- 3 fatty acids replaced mainly the omega- 6 fatty acids: linoleic acid (18:2, n-6), $\gamma$-linolenic acid (18:3, n-6), and dihomo- $\gamma$-linolenic acid $(20: 3, n-6)$. The amount of arachidonic acid $(20: 4, n-6)$, however, significantly increased.

Figure 2 shows the differences between incorporation of relative amounts of selected fatty acids in the plasma cholesterol ester fraction and in the total phospholipid and sphingolipid fraction of isolated neutrophils before and during 12 weeks of fish oil supplementation. Owing to technical difficulties the fatty acid composition of neutrophil membrane phospholipids was calculated for only nine patients. In both fractions $20: 5, \mathrm{n}-3$ was signifi-

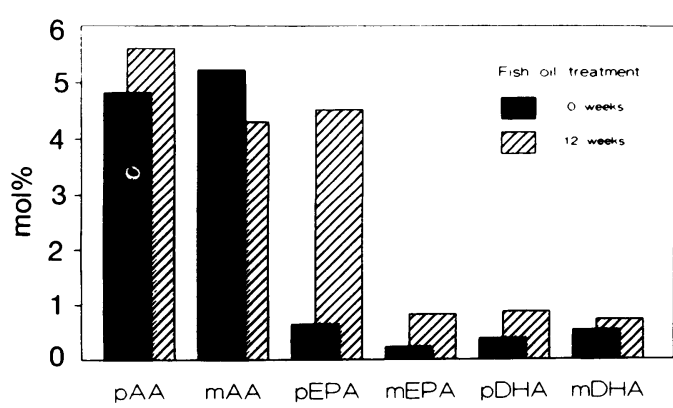

Figure 2: Differences between incorporation of relative amounts (molll) of selected fatty acids in the plasma cholesterol ester fraction and in the total phospholipid plus sphingolipid fraction isolated from neutrophils before and after three months of fish oil supplementation. Values are expressed as means. $p=$ plasma cholesterol ester fraction; $m=$ neutrophil membrane phospholipid plus sphingolipid fraction; $A A=$ arachidonic acid; $E P A=$ eicosapentaenoic acid; DHA=docosahexaenoic acid. $\mathrm{mol} \%=\mathrm{mol} / 100 \mathrm{~mol}$ fatty acids. 
cantly incorporated $(p<0.01)$. In contrast with the increase of $22: 6, n-3$ in the plasma cholesterol ester fraction $(p<0.01)$, the increment of relative amounts of $22: 6, n-3$ in the membrane phospholipids during fish oil supplementation was not statistically significant. The $20: 4, n-6$ content in the neutrophil membrane phospholipids was also not significantly altered during fish oil supplementation.

\section{Discussion}

The management of patients with rheumatoid arthritis is difficult. A variety of drugs either alone or in combination are used with varying success to suppress symptoms or modify disease activity. In extensively treated patients the number of side effects is considerable and may disturb treatment. Doctors and patients have long been intrigued by the notion that some foods or food related products can provoke or alleviate rheumatic symptoms. ${ }^{30-35}$ It is also known that fasting has an anti-inflammatory effect in rheumatoid arthritis. ${ }^{36} 37$ A more sophisticated approach is based on the fact that alteration of the dietary fatty acid composition has been shown to modulate the production of eicosanoids. ${ }^{13-20} 3839$

Our study shows significant improvement of clinical disease variables in patients with rheumatoid arthritis during dietary fish oil supplementation. Although statistically significant improvement was only achieved for joint swelling index and duration of early morning stiffness, most clinical variables favoured fish oil over placebo. Based on subjective clinical observations it can be estimated that most patients experienced considerable relief while taking fish oil supplementation. Even more impressive was the improvement of patients' assessment of disease activity. A readily available method of assessing subjective improvement, the visual analogue pain scale, did not show statistically significant alterations, however. Subjective improvements were also seen during coconut oil treatment, and therefore we conclude that the subjective alleviations are at least partially due to a placebo effect.

In contrast with the clinical disease variables, the changes in the laboratory indices of inflammation were not statistically significant. Our findings are in agreement with the results of other clinical studies on dietary omega-3 fatty acid supplementation in rheumatoid arthritis. ${ }^{20} 3839 \mathrm{~A}$ recent report, ${ }^{40}$ however, showed a significant decrease of mean plasma fibrinogen concentrations during a six week

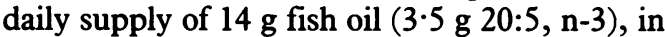
healthy volunteers. In our trial $6 \mathrm{~g}$ fish oil $(2.04$ g 20:5, n-3) had no influence of statistical importance either on the mean plasma fibrinogen concentration or on the plasma concentration of other acute phase proteins like $\mathrm{C}$ reactive protein and serum amyloid A. A possible explanation for these apparently conflicting findings may be that fibrinogen synthesis is dependent on the dose of fish oil.

Despite the unchanged concentrations of acute phase proteins, fish oil supplementation results in changes in the leucotriene production of in vitro stimulated neutrophils. A small production of leucotriene $\mathrm{B}_{5}$ was observed, concomitant with a decrease of the mean leucotriene $\mathrm{B}_{4}$ production. The slight production of leucotriene $B_{5}$ is in accordance with the small amounts of 20:5, n-3 incorporated in the total phospholipid and sphingolipid fraction from isolated neutrophils. A major uncertainty is whether any of the induced alterations of leucotriene contribute to the result of clinical improvement during fish oil treatment. As no correlations were found between changes in the leucotriene production and the significantly altered disease variables it seems unlikely that inhibition of the lipoxygenase pathway is the only cause of clinical improvement. As fish oil modulates the formation of leucotrienes, prostacyclin, and prostaglandins it is possible that the effects of fish oil may be a result of changes in the production of the prostaglandins. It is interesting to note, however, that even during the use of non-steroidal anti-inflammatory drugs, which block prostaglandin synthesis, fish oil provides an additive beneficial effect.

In conclusion this study shows that administration of omega- 3 fatty acids is effective in suppressing clinical symptoms of rheumatoid arthritis. Convincing biochemical evidence that omega-3 fatty acids may also act as a disease modifying drug were not obtained, however. Although a change in neutrophil leucotriene production in vitro could be clearly shown, the question remains whether the clinical effects can be accounted for by inhibition of the lipoxygenase pathway.

This study was supported by the Dutch League against Rheumatism. The authors wish to thank $W$ Wassenaar for nursing assistance; $M$ Strubbe and I Boomgaardt for dietary assistance; K Kazemier, G Meiborg, H M Rutgers, and I A Martini for thei valuable technical assistance, and Drs $M$ A van Leeuwen and B Nienhuis, rheumatologists, for medical advice.

1 Borgeat P, Samuelsson B. Arachidonic acid metabolism in polymorphonuclear leukocytes: effects of ionophore A23187. Proc Natl Acad Sci USA 1979; 76: 2148-52.

2 Zijlstra F J, Naaktgeboren M, Mons H, Vincent J E. Formation of prostaglandins and leukotrienes by human lung tissue in vitro after activation by the calcium ionophore A23187. Eur 7 Clin Invest 1987; 17: 325-9.

3 Malmsten C L. Prostaglandins, thromboxanes, and leukotrienes in inflammation. Semin Arthritis Rheum 1985; 15 (suppl 1): 29-35.

4 Samuelsson B, Dahlen S E, Lindgren J A, Rouzer C A Serhan C N. Leukotrienes and lipoxines: structures, biosynthesis, and biological effects. Science 1987; 237: 1171-5.

5 Samuelsson B. Leukotrienes: mediators of immediate hypersensitivity reactions and inflammation. Science 1983; 220 568-75.

6 Parker C W. 5-Lipoxygenase, leukotrienes and regulation of inflammatory responses. Drug Development and Research 1987; 10: 277-93.

7 Klickstein L B, Shapleigh C, Goetzl E J. Lipooxygenation of arachidonic acid as a source of polymorphonuclear leukocyte chemotactic factors in synovial fluid and tissue in rheumatoid arthritis and spondyloarthritis. 7 Clin Invest 1980; 66: 1166-70.

8 Ford-Hutchinson A W, Bray M A, Doig M V, Shipley M E Smith M J H. Leukotriene B, a chemokinetic and aggregating substance released from polymorphonuclear leukocytes. Nature 1980; 286: 264-5.

9 Elmgreen J, Nielsen O H, Ahnfelt-Rønne I. Enhanced capacity for release of leucotriene $B_{4}$ by neutrophils in rheumatoid arthritis. Ann Rheum Dis 1987; 46: 501-5.

10 Hoover R L, Karnovsky M J, Austen K F, Corey E J, Lewis R A. Leukotriene B4 action on endothelium mediate augmented neutrophil/endothelial adhesion. Proc Natl Acad Sci USA 1984; 81: 2191-3.

11 Palmer R M J, Stepney R J, Higgs G A, Eakins K E Chemokinetic activity of arachidonic acid lipoxygenase products on leukocytes of different species. Prostaglandins 1980; 2: 411-8.

12 Bray M A, Ford-Hutchinson A W, Smith M J H. Leukotrien B4: an inflammatory mediator in vivo. Prostaglandins 1981; 22. 213-22. 
13 Higgs $G$ A. The effects of dietary intake of essential fatty acids on prostaglandin and leukotriene synthesis. Proc Nutr Soc 1985 ; 44: 181-7.

14 Kelley V E, Ferretti A, Izui S, Strom T B. A fish oil diet rich in eicosapentaenoic acid reduces cyclooxygenase metabolites and suppresses lupus in mrl-lpr mice. f Immunol 1985; 3: and suppre

15 Strasser T, Fisher S, Weber P C. Leukotriene B5 is formed in human neutrophils after dietary supplementation with icosapentaenoic acid. Proc Natl Acad Sci USA 1985; 82: $1540-3$.

16 Terano T, Salmon J A, Moncado S. Biosynthesis and biological activity of leukotriene B5. Prostaglandins 1984 27:217-32

17 Lee T H, Mencia-Huerta J M, Shih C, Corey E J, Lewis R A, Austen K F. Characterization and biologic properties of 5,12-dihydroxy derivatives of eicosapentaenoic acid, including leukotriene $\mathrm{B} 5$ and the double lipoxygenase product. $\mathcal{F}$ Biol Chem 1984; 4: 2383-9.

18 Lee T H, Hoover R L, Williams J D, et al. Effect of dietary enrichment with eicosapentaenoic and docosahexaenoic acids on in vitro neutrophil and monocyte leukotrien generation and neutrophil function. $N$ Engl f Med 1985; 312: 1217-21

19 Payan D G, Wong M Y S, Chernov-Rogan T, et al. Alterations in human leukocyte function induced by ingestion of eicosapentaenoic acid. 7 Clin Immunol 1986; 6: 402-10.

20 Sperling I, Weinblatt M, Robin J L, et al. Effects of dietary supplementation with marine fish oil on leukocyte lipid mediator generation and function in rheumatoid arthritis. Arthritis Rheum 1987; 30: 988-97.

21 Leslie C A, Conte J M, Hayes K C, Cathcart E S. A fish oil diet reduces the severity of collagen induced arthritis after diet reduces the severity of collagen induced arthritis after

22 onset of the disease. Clin Exp Immunol 1988; 73: 328-32. Verhagen J, Walstra P, Veldink G A, Vliegenthart J F G,
Bruynzeel P L B. Separation and quantitation of leukotrienes by reversed phase high performance liquid chromatography. Prostaglandins Leukotrienes Med 1984; 13: 15-20.

23 Steege vander G, Muskiet F A J, Martini I A, Hutter N H Boersma E R. Simultaneous quantification of total mediumand long chain fatty acids in human milk by capillary gas chromatography with split injection. $\mathcal{f}$ Chromatogr 1987 415: $1-11$.

24 Hoving E B, Jansen G, Volmer M, Doormaal van J J, Muskiet F A J. Profiling of plasma cholesterol ester and triglyceride fatty acids as their methyl esters by capillary gas chromatography, preceded by a separation of lipid classes. $f$ graphy, preceded by a separati
25 Zijlstra J G, Vries de J G, Muskiet F A J, Martini I A, Timmer-Boscha $\mathrm{H}$, Mulder $\mathrm{N} \mathrm{H}$. Influence of docosahexaenoic acid in vitro on intracellular adriamycin-sensitive and -resistant small-cell lung cancer cell lines, and on cytotoxicity in the tumor cell lines. Int $f$ Cancer 1987; 40: $850-6$.

26 Hoving E B, Prins J, Rutgers H M, Muskiet F A J Behaviour of plasmalogens during high performance liquid chromatography on a silica collumn and a phosphoric acid containing mobile phase. $\mathcal{F}$ Chromatogr 1988; 434: 411-6.

27 Muskiet F A J, Doormaal van J J, Martini I A, Wolthers B G Slik van der W. Capillary gas chromatographic profiling of total long chain fatty acids and cholesterol in biological materials. 7 Chromatogr 1983; 278: 231-44.

28 Pocock S J. Clinical trial. New York: Wiley, 1983: 110-22.

29 Edginton E S. Randomisation tests. New York: Marcel Dekker, 1980: 101-30.

30 Anonymous. Fish oils in rheumatoid arthritis [Editorial]. Lancet 1987; i: $720-1$.

31 Panush R S, Stroud R M, Webster E M. Food induced (allergic) arthritis, inflammatory arthritis exacerbated by milk. Arthritis Rheum 1986; 29: 220-6.

32 Beri D, Malaviya A N, Shandilya R, Singh R R. Effect of dietary restrictions on disease activity in rheumatoid arthritis. Ann Rheum Dis 1988; 47: 69-72.

33 Ziff $M$. Diet in the treatment of rheumatoid arthritis. Arthritis Rheum 1983; 26: 457-71.

34 Anonymous. Nutritional therapy for rheumatic diseases [Editorial]. Ann Intern Med 1987; 4: 619-20.

35 Parje A L, Hughes G R V. Rheumatoid arthritis and food. $B$ Med F 1981; 282: 2027-9.

36 Hafstrom I, Ringertz B, Gyllenhammar H, Palmblad J Harms-Ringdal $M$. Effects of fasting on disease activity, neutrophil function, fatty acid composition, and leukotriene biosynthesis in patients with rheumatoid arthritis. Arthriti Rheum 1988; 31: 585-92

37 Uden A M, Trang L, Venizelos N, Palmblad J. Neutrophil functions and clinical performance after total fasting in patients with rheumatoid arthritis. Ann Rheum Dis 1983; 42: $45-51$

38 Kremer J M, Bigaouette J, Michalek A V, et al. Effects of manipulation of dietary fatty acids on clinical manifestations of rheumatoid arthritis. Lancet 1985; i: 184-7.

39 Kremer J M, Jubiz W, Michalek A V, et al. Fish oil fatty acid supplementation in active rheumatoid arthritis. Ann Intern Med 1987; 106: 497-503.

40 Hostmark A T, Bjerkedal T, Kierulf P, Flaten H, Ulshagen K. Fish oil and plasma fibrinogen. Br Med f 1988; 297 : 180. 